\title{
Ethics in Research
}

We don't have exact data as how many researches are going in Nepal nor we know whether they have abided by the ethics in research or not. The clinical trials show that four researches are recruiting participants and 13 have been completed when searched for the term Nepal. ${ }^{1}$ There has been lots of research going on lately in the country partly due to increasing awareness and/or for academic achievement. Since the study is done under pressure, it bounds to violate the ethics.

The culture of research and reporting has not been developed yet. But we believe this is the right time to sensitize all the researchers and author about ethics in research. Ethics or morals are neither about what is right or wrong, legal or illegal nor like Ten Commandments. An action may be legal but unethical or illegal but ethical.

When most people think of ethics (or morals), they think of rules for distinguishing between right and wrong, such as the Golden Rule, a code of professional conduct like the Hippocratic Oath or a wise aphorisms like the sayings of Confucius. This is the most common way of defining "ethics": ethics are norms for conduct that distinguish between or acceptable and unacceptable behavior. ${ }^{2}$

Most people learn ethical norms at home, at school, in church, or in other social settings. Although most people acquire their sense of right and wrong during childhood, moral development occurs throughout life and human beings pass through different stages of growth as they mature. Ethical norms are so ubiquitous that one might be tempted to regard them as simple commonsense. ${ }^{2}$

Most societies also have legal rules that govern behavior, but ethical norms tend to be broader and more informal than laws. Although most societies use laws to enforce widely accepted moral standards and ethical and legal rules use similar concepts, it is important to remember that ethics and law are not the same. We can also use ethical concepts and principles to criticize, evaluate, propose, or interpret laws. Indeed, in the last century, many social reformers urged citizens to disobey laws in order to protest what they regarded as immoral or unjust laws. ${ }^{2}$

The Ethical Review Board at Nepal Health Research Council looks after the ethics in research in Nepal. ${ }^{3-6}$ It recognized Institutional Review Committee in 14 institutes and organizations throughout the country and is expanding more in the future. The researcher has to apply for the approval before a study is initiated. The researchers are encouraged to take the approval from their own institutional IRC if it is not available contact ERB. Most of the general researches are approved by IRC but some research need approval from ERB.

In the scenario of increasing awareness about knowledge and consumer protection Journal of Nepal Health Research Council has decided to foster ethical practice in research and reporting a study. The JNHRC will accept only those research papers with ethical approval letter, submitted after January, 2010.

\section{REFERENCES}

1. Clinical Trials. Found 17 studies with search of: Nepal. [cited 2009 Set 15]; Available from: http://clinicaltrials.gov/ct2/ results?term $=$ nepal.

2. Resnik D. What is Ethics in Research \& Why is It Important? : National Instituet of Environmental Health Sciences - National Institute of Health; [cited 2009 Sep 15]; Available from: http://www.niehs.nih.gov/research/resources/bioethics/whatis.cfm.

3. Nepal Health Research Council. National ethical guidelines for health research in Nepal. Kathmandu: Nepal Health Research Council; 2001.

4. Nepal Health Research Council. National Ethical guidelines on clinical trails with the use of pharmaceutical products . Kathmandu: Nepal Health Research Council; 2005.

5. Nepal Health Research Council. Ethical guidelines for the care and use of animals in health research in Nepal. Kathmandu: Nepal Health Research Council; 2005.

6. Nepal Health Research Council. Guidelines for institutional review committees for health research in Nepal. Kathmandu: Nepal Health Research Council; 2001. 